\title{
Prevalence of Aminoglycoside Resistance Genes in Acinetobacter baumannii Isolates
}

\author{
Katayun Aliakbarzade ${ }^{1}$; Safar Farajnia ${ }^{2, *}$; Ashraf Karimi Nik ${ }^{1}$; Farzaneh Zarei ${ }^{3}$; Asghar \\ Tanomand $^{4}$ \\ ${ }^{1}$ Department of Microbiology, Science and Research Branch, Islamic Azad University of Kerman, IR Iran \\ ${ }_{2}^{2}$ Drug Applied Research Center, Tabriz University of Medical Sciences, Tabriz, IR Iran \\ ${ }^{3}$ Biotechnology Research Center, Tabriz University of Medical Sciences, Tabriz, IR Iran \\ ${ }^{4}$ Tuberculosis and Lung Research Center, Tabriz University of Medical Sciences, Tabriz, IR Iran \\ ${ }^{*}$ Corresponding author: Safar Farajnia, Drug Applied Research Center, Tabriz University of Medical Sciences, Tabriz, IR Iran. Tel: +98 -9143018589 Fax: +98-4113363231, E-mail: faraj- \\ nias@tbzmed.ac.ir
}

Received: June 09, 2013; Revised: Augest 05, 2013; Accepted: September 10, 2013

\begin{abstract}
Background: Acinetobacter baumannii is one of the major causes of nosocomial infections and is resistant to most available antibiotics. Aminoglycosides remain as drugs of choice for treatment of Acinetobacter infections yet resistance to aminoglycosides has increased in the recent years.

Objectives: The present study investigated the prevalence of genes encoding aminoglycoside-modifying enzymes in A. baumannii strains isolated from patients of Tabriz city, northwest of Iran.

Materials and Methods: A total of 103 Acinetobacter isolates were collected from Imam Reza Hospital of Tabriz University of medical sciences. Antimicrobial susceptibility patterns of the isolates to different antimicrobial agents including cephalosporins, gentamicin, amikacin, tobramycin, colistin and polymyxin, were evaluated by the disc diffusion method. The frequency of aminoglycoside modifying enzymes encoding genes aacC1, aphA6, aadA1 and aadB was analyzed by the PCR method.

Results: Antimicrobial susceptibility analysis showed that the highest resistance was towards beta -lactam antibiotics including cephalosporins whereas the highest sensitivity was observed towards colistin (77\%) and polymyxin (84\%). The resistance rate to aminoglycosides was $81 \%, 86 \%$ and $63 \%$ for amikacin, gentamicin and tobramycin, respectively. The PCR results showed that among the 103 A. baumannii isolates, 56 (65.11\%) were positive for aacC1, 52 (60.46\%) for aphA6, $24(27.9 \%)$ for aadA1 and 16 (18.6\%) for aadB resistant genes. Conclusions: The results of this study indicated that the genes encoding aminoglycoside-modifying enzymes are prevalent in $A$. baumannii isolates in the study region, which highlighted the necessity of considering preventive measures to control dissemination of these resistance genes.
\end{abstract}

Keywords:Acinetobacter Baumannii; Antimicrobial Agents; Aminoglycoside

\section{Background}

Acinetobacter baumannii is a Gram-negative, aerobic, non-motile, non-fastidious strictly aerobic and glucosenon-fermenting bacterium that has coccobacillary morphology on non-selective agar and is classified as an opportunistic pathogen in hospitals. This bacterium is commonly found in soil, water and sewage. It was experimentally shown that the majority of A. baumannii strains survive longer than Escherichia coli on dry surfaces, and some strains survive for more than four months. These characteristics are beneficial for the organism to survive in hospital environments and cause infection. A. baumannii has been recognized as the most important cause of nosocomial infections in immunocompromised patients particularly those in the intensive care units (ICUs) (1), and is ranked second after Pseudomonas aeruginosa among gram -negative nosocomial pathogens (2).

A. baumannii is the causative agent of several types of infections including bloodstream infections, ventilator-as- sociated pneumonia, skin and soft tissue infections, meningitis and urinary tract infections (3). This bacterium can survive on different medical equipments and even on healthy human skin (4). A. baumannii is resistant to most available antibiotics and there are increasing reports of multidrug-resistant A. baumannii (MDRAB) outbreaks in clinical settings worldwide (5). Multidrug-resistant $A$. baumannii strains are resistant to at least three different classes of antimicrobial agents mainly beta-lactams, aminoglycosides, carbapenems and fluoroquinolones (6).

Aminoglycosides show various characteristics that make them useful for antimicrobial therapy. The bactericidal activity of aminoglycosides depend more on their concentration than on the duration of bacterial exposure to inhibitory concentrations of the antibiotics. The potential of aminoglycosides to kill bacteria depends on the concentration of the antibiotic, and increases with increasing concentrations. In addition, aminoglycosides

Copyright (C) 2014, Ahvaz Jundishapur University of Medical Sciences; Published by Kowsar. This is an open-access article distributed under the terms of the Creative Commons Attribution License, which permits unrestricted use, distribution, and reproduction in any medium, provided the original work is properly cited. 
continue to kill bacteria even after the aminoglycoside is detectable, exhibiting an important post -antibiotic effect. This is probably due to a strong, irreversible binding to the ribosome. These drugs attack the bacteria in a two-step process; firstly, uptake of aminoglycosides into the bacteria takes place, which is an important process for their biological activity, and secondly, inside the bacterial cell the aminoglycoside binds to the ribosome and inhibits protein synthesis. Therefore, aminoglycosides represent an important group of antibiotics in treating different bacterial infections. However, in the recent years different resistance mechanisms have emerged against these antimicrobial agents.

Extended spectrum beta lactamases (ESBLs) are a class of group A beta lactamases which hydrolyze first, second and third generation cephalosporines but are inhibited by beta-lactamase inhibitors like clavulanic acid (7-9). The most prevalent resistance mechanisms to carbapenem antibiotics in A.baumannii is production of OXA - type $ß$-lactamases $(10,11)$, and resistance to quinolones is related to alterations in the target enzymes, GyrA and ParC. Aminoglycosides have long been used for the treatment of Acinetobacter infections and still are an important alternative for therapy of infections caused by MDR strains. However, resistance to aminoglycosides has increased in the recent years in these bacteria (12).

The major mechanism of aminoglycoside resistance in clinical isolates of gram-negative bacteria is enzymatic modification of amino- or hydroxyl-groups of the aminoglycosides. Enzymatic modification of aminoglycosides results in reduced or abolished binding of the aminoglycoside molecule to the ribosome. Previous studies indicated that there are several mechanisms of resistance to aminoglycosides in the Acinetobacter spp. $(13,14)$. The most prevalent resistance mechanism is attributed to enzymatic inactivation by acetyltransferases (AAC), nucleotidyl transferases (ANT) and (phosphotransferases) APH (13). Many of the aminoglycoside modifying enzymes (AME) results in clinical resistance, but in general only the APHs and AACs produce high levels of resistance.

\section{Objectives}

The aim of this study was to evaluate the prevalence of aminoglycoside resistance and frequency of aacC1, aphA6, aadA1 and aadB aminoglycoside resistance genes among A. baumannii strains isolated from patients referred to Imam Reza hospital of Tabriz city.

\section{Materials and Methods}

\subsection{Bacterial Isolation and Identification}

In this study a total of 103 clinical A. baumannii strains were collected from hospitalized patients at Imam Reza Hospital of Tabriz University of Medical Sciences. The isolates were from different clinical samples including tra- cheal secretion, bronchial lavage, blood, wound, sputum and urine. The samples were transferred to the laboratory of the Department of Microbiology in the medicine faculty and all isolates were identified by using standard biochemical tests such as, Gram staining, oxidase test, catalase test, motility, citrate utilization, oxidative/fermentative glucose (O/F) test and growth ability at $44^{\circ} \mathrm{C}$ (15). Species identification was confirmed by detection of blaOXA-51-like genes, as described previously (16).

\subsection{Antimicrobial Susceptibility Test}

The antimicrobial resistance of the isolates was determined by using the standard disk diffusion method according to the Clinical Laboratory Standard Institute (CLSI) guidelines (2011). Disk diffusion is a semi quantitative method used to examine bacterial susceptibility to specific antibiotics. It allows categorization of bacterial isolates as susceptible, intermediate or resistant to a variety of antimicrobial agents. The antimicrobial agents used in this study consisted of cefotaxime $(30 \mu \mathrm{g})$, cefixime $(5 \mu \mathrm{g})$, ceftizoxime (30 $\mu \mathrm{g})$, ceftazidime (30 $\mu \mathrm{g})$, ceftriaxone $(30 \mu \mathrm{g})$, cephalexin $(30 \mu \mathrm{g})$, cephalothin $(30 \mu \mathrm{g})$, tetracycline $(30 \mu \mathrm{g})$, ciprofloxacin $(5 \mu \mathrm{g})$, cotrimoxazole $(25 \mu \mathrm{g})$, ticarcillin $(75 \mathrm{mg})$, polymyxin B $(300 \mu \mathrm{g})$, tobramycin $(10 \mu \mathrm{g})$, chloramphenicol $(30 \mu \mathrm{g})$, norfloxacin (10 $\mu \mathrm{g})$, ofloxacin $(5 \mu \mathrm{g})$, amikacin $(30 \mu \mathrm{g})$, gentamicin $(10 \mu \mathrm{g})$, ampicillin $(10 \mu \mathrm{g})$, carbenicillin $(100 \mu \mathrm{g})$, rifampin $(5 \mu \mathrm{g})$ and colistin $(110 \mu \mathrm{g})($ MAST, Merseyside, UK). The isolates were cultured on Mueller Hinton Agar plates (Merck, Germany) inoculated with a bacterial suspension equal to $0.5 \mathrm{McF}$ arland and incubated at $37^{\circ} \mathrm{C}$ for $18-24$ hours. The diameter of the zone of growth inhibition was measured using the CLSI guidelines (16).

\subsection{DNA Extraction and PCR Amplification}

All A. baumannii isolates were grown for 18 hours at $37^{\circ} \mathrm{C}$ in MacConkey agar (Difco BD bioscience) and DNA were extracted by sodium dodecyl sulfate (SDS)-Proteinase K phenol chloroform method as described previously (17). Briefly, 4-5 fresh colonies were resuspended in $300 \mu \mathrm{L}$ of tris-EDTA (TE) buffer containing SDS (1\%) and proteinase $\mathrm{K}(10 \mu \mathrm{g} / \mathrm{mL})$, and incubated at $40^{\circ} \mathrm{C}$ for 3 hours followed by phenol-chloroform extraction and ethanol precipitation. Several washing steps were performed, to improve the purity of the DNA. DNA was finally eluted in water and preserved at $-20^{\circ} \mathrm{C}$ until use.

\subsection{Detection of aminoglycoside-resistance genes}

The genes encoding aminoglycoside-modifying enzymes, including phosphotransferases APH (3')-Via (aphA6), acetyltransferases AAC (3)-Ia (aacC1), nucleotidyl transferases ANT (2")-Ia (aadB) and ANT(3") -Ia (aadA1), were detected by PCR. Polymerase chain reaction (PCR) is a rapid method for in vitro amplification of DNA. The sequences of primers used in the PCR amplification are illustrated in Table 1. 
Aliakbarzade Ket al.

\begin{tabular}{|c|c|c|c|}
\hline Primer & Primer Sequences & Product Size, bp & Annealing Temperature, ${ }^{\circ} \mathrm{C}$ \\
\hline aphA6 F & ATGGAATTGCCCAATATTATTC & 797 bp & 55 \\
\hline aphA6 R & TCAATTCAATTCATCAAGTTTTA & & \\
\hline aadA1 $F$ & ATGAGGGAAGCGGTGATCG & 792 bp & 52 \\
\hline $\operatorname{aadA1} R$ & TTATTTGCCGACTACCTTGGTG & & \\
\hline $\operatorname{aadB} \mathbf{F}$ & ATGGACACAACGCAGGTCGC & 534 bp & 55 \\
\hline aadB $\mathbf{R}$ & TTAGGCCGCATATCGCGACC & & \\
\hline $\operatorname{aacC1} \mathrm{F}$ & ATGGGCATCATTCGCACATGTAGG & 456 bp & 52 \\
\hline aacC1 $R$ & TTAGGTGGCGGTACTTGGGTC & & \\
\hline
\end{tabular}

Table 2. Thermocycler Program for Amplification of Gene AphA6/ aadB/aadA1/aacC1

\begin{tabular}{lccc}
\hline NO. & Parameter Steps & Temperature, ${ }^{\circ} \mathbf{C}$ & Time \\
\hline $\mathbf{1}$ & initial denaturation & 95 & $3^{\prime}$ \\
$\mathbf{2}$ & denaturation & 94 & $1^{\prime}$ \\
$\mathbf{3}$ & annealing & $55 / 55 / 52 / 52$ & $1^{\prime}$ \\
$\mathbf{4}$ & cycle number & 35 & \\
$\mathbf{5}$ & extension & 72 & $45^{\prime \prime}$ \\
$\mathbf{6}$ & final extension & 72 & $5^{\prime}$ \\
\hline
\end{tabular}

\subsection{PCR Analysis}

PCR reactions were performed in a final volume of $25 \mu \mathrm{L}$ $(24 \mu \mathrm{L}$ PCR master mix plus $1 \mu \mathrm{L}$ of template DNA). PCR amplification was carried out in a thermal cycler with the following parameters: initial denaturation at $95^{\circ} \mathrm{C}$ for $3 \mathrm{~min}$ utes, followed by 35 cycles of 60 seconds, denaturation at $94^{\circ} \mathrm{C}, 60$ seconds for annealing at primers annealing temperatures (Table 1 ) and 45 seconds for extension at $72^{\circ} \mathrm{C}$ with a final extension at $72^{\circ} \mathrm{C}$ for 5 minutes (Table 2). The presence and sizes of amplicons were analyzed by electrophoresis on $1.2 \%$ agarose gel in a tris-acetate-EDTA (TAE) buffer at 90 volts alongside a $1 \mathrm{~Kb}$ DNA ladder (Fermentas, Lithuania). Electrophoresis was performed for one hour and finally the gels were stained with ethidium bromide and visualized using the gel documentation system.

\section{Results}

\subsection{Characteristics of the Study Population}

The mean age of the population was 51 years, with a range of 14 to 86 years. The isolates were obtained from patients belonging to different age groups:20 -39 years $(n=26), 40-59(n=40), 60-90$ years $(n=34)$ and three isolates were from patients less than 20 years old.

\subsection{Isolates and Antimicrobial Susceptibility}

A total of 103 A. baumannii isolates were recovered from clinical specimens of hospitalized patients that had been admitted to the Imam Reza Hospital of Tabriz, North-West of Iran. The isolates were obtained from invasive and noninvasive sites, including trachea (38\%), urine (22\%), sputum (10\%), blood (7\%), catheter (6\%), bronchial washings
Table 3. Antimicrobial Resistance Pattern of Acinetobacter Baumannii Against Different Antibiotics

\begin{tabular}{lccc}
\hline Antibiotics & Resistant,\% Intermediate, \% Sensitive, \% \\
\hline Ticarcillin & 100 & 0 & 0 \\
Cefixime & 100 & 0 & 0 \\
\hline Ceftizoxime & 100 & 0 & 0 \\
\hline Cephalexin & 97 & 0 & 3 \\
\hline Cefotaxime & 97 & 0 & 3 \\
\hline Ampicillin & 94 & 0 & 6 \\
\hline Ceftriaxone & 94 & 0 & 6 \\
\hline Ceftazidime & 93 & 0 & 7 \\
\hline Cephalothin & 91 & 0 & 9 \\
\hline Carbenicillin & 89 & 0 & 11 \\
\hline Gentamicin & 86 & 5 & 9 \\
\hline Cotrimoxazole & 85 & 0 & 15 \\
\hline Norfloxacin & 84 & 0 & 16 \\
\hline Amikacin & 81 & 2 & 17 \\
\hline Ciprofloxacin & 80 & 0 & 20 \\
\hline Chloramphenicol & 78 & 0 & 22 \\
\hline Ofloxacin & 71 & 0 & 29 \\
\hline Tetracycline & 65 & 31 & 4 \\
\hline Tobramycin & 63 & 0 & 37 \\
\hline Rifampin & 27 & 0 & 73 \\
\hline Colistin & 19 & 4 & 0 \\
\hline Polymyxin B & 16 & & 0 \\
\hline & & 0 & 0 \\
\hline
\end{tabular}

(6\%), wound (5\%), abscess drainage (3\%), cerebrospinal fluid (2\%), ascites fluid (2\%) and pleural effusion (2\%). The samples were transferred to the laboratory of the Microbiology Department of Tabriz University and were immediately inoculated on to MacConkey and blood agar plates. Analysis of antimicrobial susceptibility in this study showed that the highest resistance was against cefiexime (100\%), ceftizoxime (100\%) and ticaracilin (100\%), whereas the highest susceptibility was observed towards polymyxin B (84\%), colistin (77\%) and rifampin (73\%) (Table 3). Resistance to various aminoglycosides was as follows; gentamicin $86 \%$, tobramycin $63 \%$ and amikacin $81 \%$. Tobramycin was the most active agent tested. 
Figure 1. Amplification of aacC1 Resistance Gene by the PCR Technique

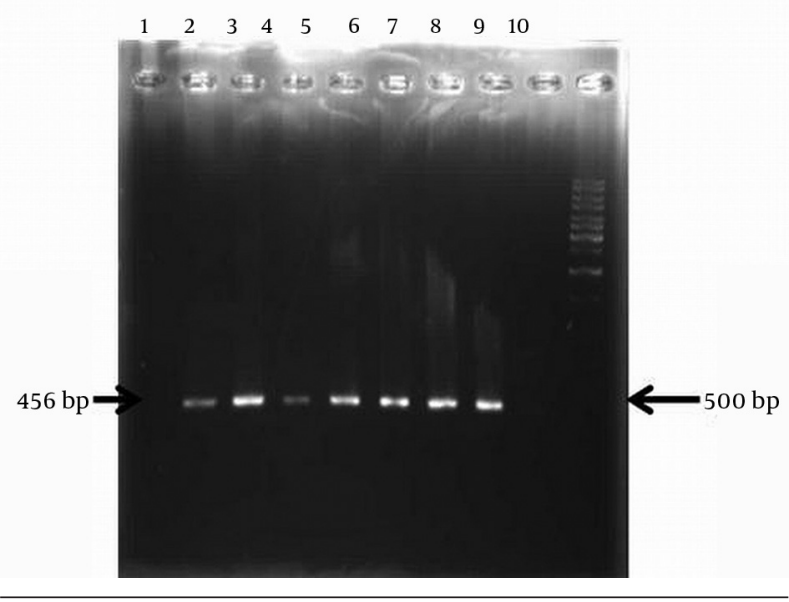

Lane 1 isolate negative for aacC1 gene, Lane 2-8 isolates with aacC1 gene, Lane 9, no DNA and Lane 10 related to size marker (1 kb DNA ladder).

Figure 2. Amplification of aphA6 Resistance Gene by the PCR Technique

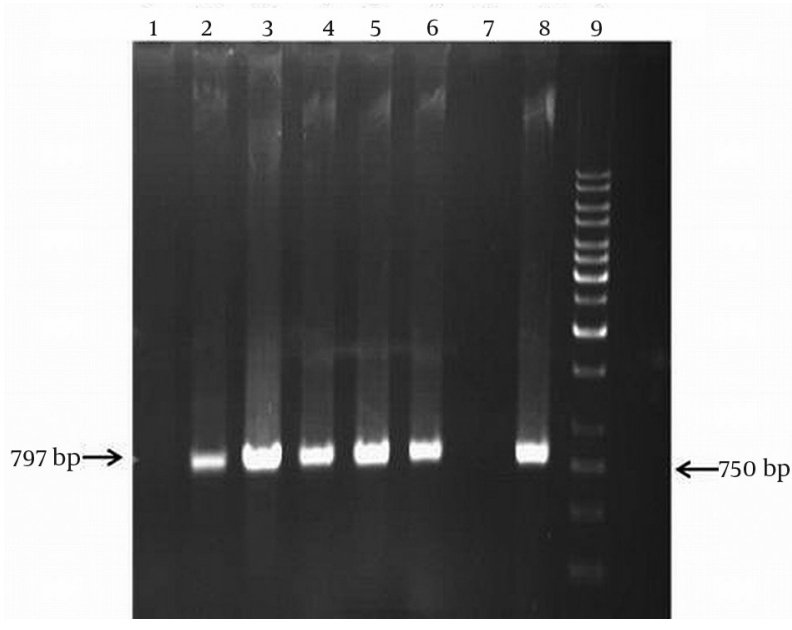

Lane 1 and 7, isolates negative for aphA (6) gene, Lane 2_6 and 8, isolates with aphA (6) genes, Lane 9, size marker (1 kb DNA ladder).

\subsection{Aminoglycoside-Modifying Enzymes}

Screening of aminoglycoside resistant isolates for resistance genes by PCR (Figure $1-4$ ) revealed that the prevalence of the aacC1 gene, which confers resistance to amikacin and gentamicin, was $65.11 \%$. Screening for aphA6, aadA1and aadB genes revealed that $52(60.46 \%)$ isolates were positive for aphA6, 24 (27.9\%) for aadA1 and 16 (18.6 \%) for aadB, respectively.

Twenty one resistant isolates (20.4\%) had no AME genes. Isolates with two AME genes were seen in 32 (31.06\%) cases for aacC1 and aphA6, 17 (16.5\%) cases for aadA1 and aphA6 genes, 15 (14.56\%) cases for aacC1 and aadA1, 10 (9.7\%) cases for aphA6 and aadB, 8 (7.76\%) cases for aadA1 and aadB genes and 6 (5.82\%) isolates had aacC1 and aadB genes.

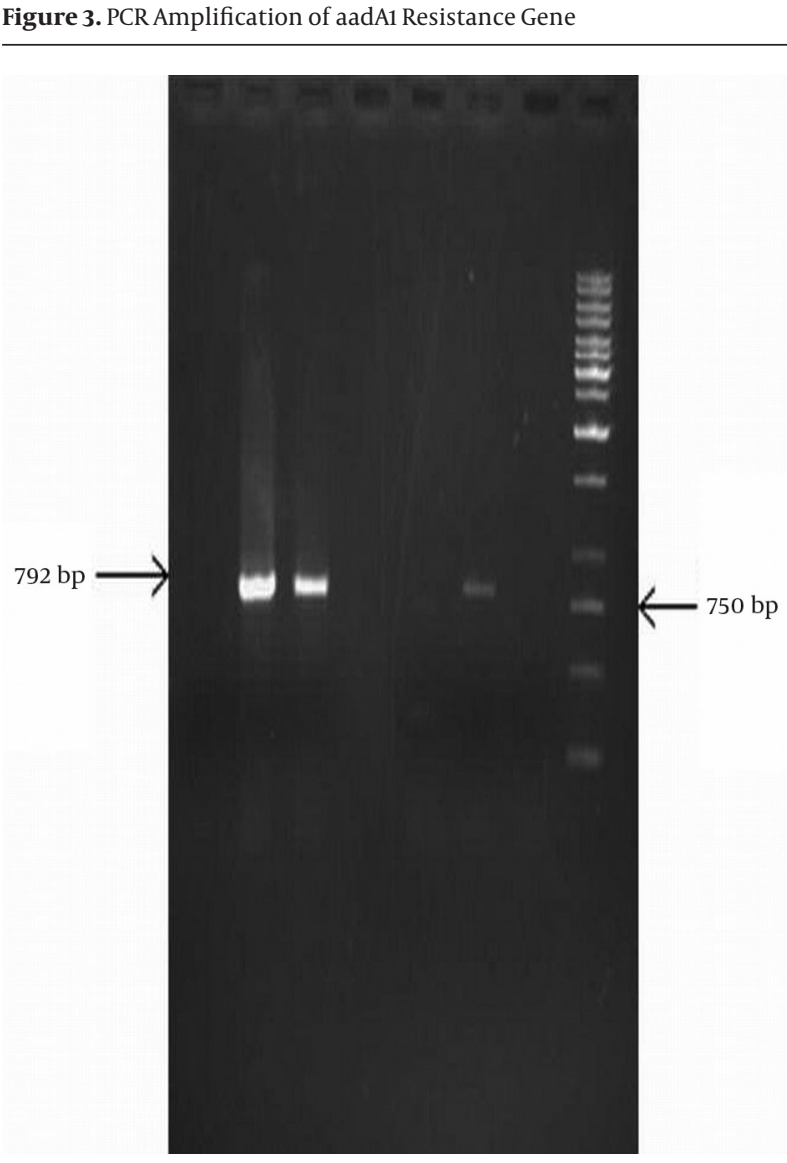

Lane 1,4,5 isolates negative for aadA1 gene, Lane 2, 3 and 6, isolates with aadA1 gene, Lane 7, no DNA and Lane 8 related to size marker (1 kb DNA ladder).

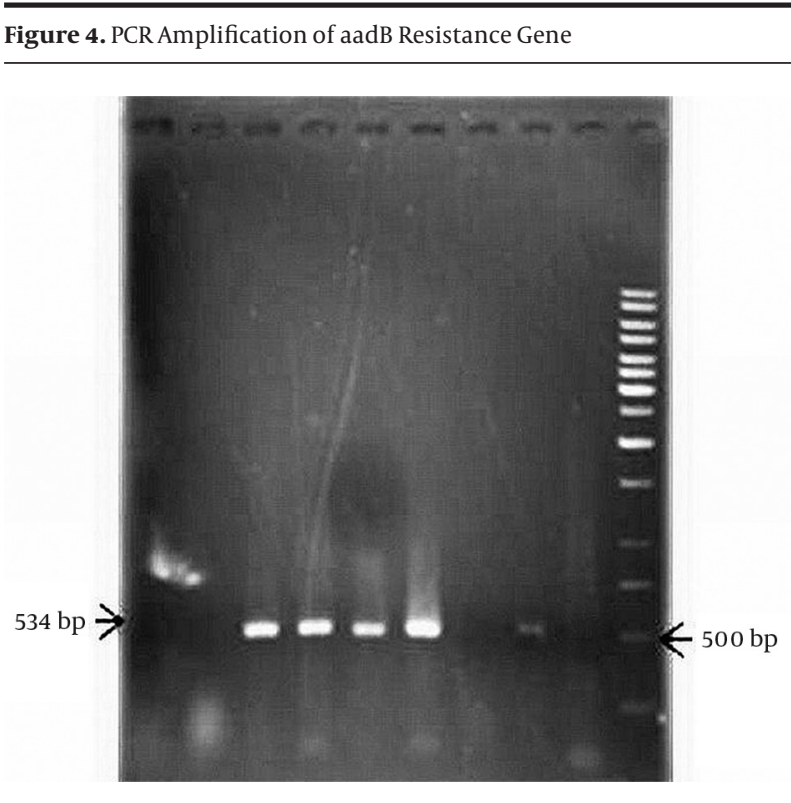

Lane 1, 2, 7 and 8 isolates negative for aadB gene, Lane 3, 4, 5 and 6 isolates with aadB gene, Lane 9 , no DNA and Lane 10 size marker (1 kb DNA ladder). 
Nine isolates (8.73\%) had three AME genes, aacC1, aadA1 and aphA6. Three isolates were positive for aacC1, aphA6 and $\mathrm{aadB}$, and three isolates had a combination of four different AME genes including aacC1, aphA6, aadB and aadA1. Strains with aacC1 or aphA6 were found to be resistant to kanamycin and gentamicin or kanamycin and amikacin, respectively, while aadB was associated with resistance to kanamycin, gentamicin and tobramycin (18).

\section{Discussion}

MDR A. baumannii is an important pathogen that is involved in nosocomial infections especially in ICU wards. This bacterium is one of the most important problems encountered in hospitals, clinics and public health centers (19). This organism is very difficult to eradicate due to its inherent and acquired resistance against multiple classes of antibiotics, so that very few effective therapeutic options remain available. Aminoglycosides have been an important group of antibiotics in treatment of serious bacterial infections, especially those with aerobic Gram negative bacteria, but recent reports indicated the emergence of resistance to aminoglycosides in Acinetobacter isolates in different parts of the world. Aminoglycoside resistance in Acinetobacter primarily results from inactivation of the antibiotic by specific modifying enzymes such as acetyl transferases, phosphotransferases, and adenylyl transferases.

This study focused on resistance to different aminoglycosides in clinically important isolates of A. baumannii, with emphasis on gentamicin, tobramycin and amikacin. The prevalence of AMEs encoding genes were investigated in A. baumannii isolates recovered from patients hospitalized in Tabriz city in the North West of Iran. Findings of the present study showed that $65.11 \%$ of the studied Acinetobacter isolates were positive for aacC1 genes. This indicates a high prevalence of resistance due to aacC1 gene in the studied cases. Also otheraminoglycoside -modifying enzyme genes detected by PCR were aphA6 (60.46\%), aadA1 $(27.9 \%)$ and aadB (18.6 \%). These results indicate that the rate of resistance by aminoglycoside-modifying enzyme types aphA6, aadA1 and aadB have increased significantly over the past years.

Moniri et al. (20) evaluated antimicrobial susceptibility and aminoglycoside resistance genes of sixty Acinetobacter strains isolated from hospitalized patients in Kashan city. They reported the presence of acetyltransferase genes (aacC1) in 63.3\% of Acinetobacter isolates. Other genes including phosphotransferase (aphA6) and adenylyl transferase (aadA1 and aadB) were detected in $65 \%, 41.7 \%$ and $3.3 \%$, of the isolates, respectively. In our study the prevalence of aphA6 and aadA1 genes was much lower than that reported from Kashan city but the prevalence of aadB genes was significantly higher in our study. These findings showed that clinical isolates of Acinetobacter in hospitals carry various kinds of aminoglycoside resistance genes. Also, Acinetobacter isolates in the study of Moniri et al. from Kashan city showed the highest resis- tance rate against amikacin, tobramycin and ceftazidim, respectively; while isolated bacteria were more sensitive to ampicillic/subactam. The resistance rates reported for amikacin and tobramycin were $80 \%$ and $68.3 \%$, respectively. There is little difference between our findings and this study. In our study the resistance rate to amikacin was $81 \%$ and tobramycin $63 \%$. Another study in Iran was done by Shahcheraghi and his colleagues in Tehran (21), which showed the highest resistance (100\%) to cefixime that was very similar to our study. The lowest resistance was reported against colistin (4.2\%) whereas in our study, $19 \%$ of isolates were resistant to colistin (21).

In a study done by Lee and his colleagues in Korea in 2011 (12), the majority of aminoglycoside-modifying enzyme genes detected by PCR were aacC1 (56\%), aadB (48\%), and aphA6 (71\%) (12). Nemec and his colleagues in Czech Republic in 2004 investigated the diversity of genes encoding aminoglycoside -modifying enzymes and their association with class 1 integrons in A. baumannii and reported aminoglycoside resistance genes in $95 \%$ of isolates: aacC1 $(n=68)$, aadA1 $(n=68)$, aphA6 $(n=55)$, and $\operatorname{aadB}(\mathrm{n}=31)(18)$. The rate of resistance in our study was considerably higher than the rates found for other geographical regions including Korea and Czech Republic.

Akers et al. (22) in 2010 studied the susceptibility of 107 isolates of A. baumannii-calcoaceticus complex to amikacin, gentamicin and tobramycin using the disk diffusion method. The susceptibility to aminoglycoside antibiotics were reported $96.6 \%$ to gentamicin and $77.5 \%$ to tobramycin. In the Akers study, $56.1 \%$ of isolates contained two and 3.7\% contained three AME genes. In our study resistance to gentamicin and tobramycin were $86 \%$ and $63 \%$, respectively. In the present study, isolates with two AME genes were seen in 32 (31.06\%) cases for aacC1 and aphA6; 17 (16.5\%) cases for aadA1 and aphA6 genes; 15 (14.56\%) cases for aacC1 and aadA1; 10 (9.7\%) cases for aphA6 and aadB; 8 (7.76\%) cases for aadA1 and aadB genes and 6 (5.82\%) isolates had aacC1 and aadB genes. These results are in agreement with some other studies that have found that aacC1 and aphA6 genes are the most common AME genes in A. baumannii isolates. Lee et al. and Moniri et al. $(12,20)$ detected aacC1 in $56 \%$ and $63.3 \%$ of isolates, respectively. In another study that was done by Nigro et al. (23) in Australia they investigated the pattern of resistance to aminoglycosides in sixty -one multi-resistant A. baumannii strains isolated between 2000 and 2010 in six Australian hospitals. In this work the isolates were screened for AME genes; aadB, aacC1, aphA1b, aphA6 and OXA 23 beta-lactamase gene (20). They found that the aphA6 gene was present in combination with aacC1 and aphA1 in two isolates.

Our study results showed a remarkable diversity of genes encoding aminoglycoside -modifying enzymes in the study region. The multiple resistance mechanisms in A. baumannii isolates make this bacterium a major clinical and public health concern. Resistance of these bacteria to commercially available drugs subsequently makes their therapy extremely difficult. Our study results indi- 
cated that the genes related to AME are prevalent in the $A$. baumannii strains in the study region which highlighted the necessity of considering preventive measure to control dissemination of resistance genes (21).

\section{Acknowledgements}

The authors like to thank Dr. Paymani and Dr. Sohrabi for their technical assistance and providing some of isolates for this work.

\section{Authors' Contributions}

Study concept and design: Dr. Safar Farajnia. Performing experiments: Katayun Aliakbarzade. Analysis and interpretation of data: Dr. Safar Farajnia. Drafting of the manuscript: Katayun Aliakbarzade and Safar Farajnia. Critical revision of the manuscript: Safar Farajnia and Ashraf Karimi Nik. Statistical analysis: Asghar Tanomand and Farzaneh Zarei.

\section{Funding/Support}

This study was supported in part by a grant from the research deputy of Tabriz University of Medical Sciences.

\section{References}

1. Dinc U, Bayramoglu G, Buruk K, Ulusoy H, Tosun I, Kaklikkaya N. Molecular epidemiology of Acinetobacter baumannii-Acinetobacter calcoaceticus complex isolated from clinical specimens at an intensive care unit. Saudi Med J. 2010;31(4):453-5.

2. Berlau J, Aucken H, Malnick H, Pitt T. Distribution of Acinetobacter species on skin of healthy humans. Eur J Clin Microbiol Infect Dis. 1999;18(3):179-83.

3. Fournier PE, Richet H. The epidemiology and control of Acinetobacter baumannii in health care facilities. Clin Infect Dis. 2006;42(5):692-9.

4. Murray PR, Baron EJ. Manual of Clinical Microbiology.washington: ASM Press; 2007.

5. Peleg AY, Seifert H, Paterson DL. Acinetobacter baumannii: emergence of a successful pathogen. Clin Microbiol Rev. 2008; 21(3):538-82.

6. Huang LY, Chen TL, Lu PL, Tsai CA, Cho WL, Chang FY, et al. Dissemination of multidrug-resistant, class 1 integron-carrying Acinetobacter baumannii isolates in Taiwan. Clin Microbiol Infect. 2008;14(11):1010-9.

7. Bradford PA. Extended-spectrum beta-lactamases in the 21st century: characterization, epidemiology, and detection of this important resistance threat. Clin Microbiol Rev. 2001;14(4):933-51.

8. Jacoby GA, Munoz-Price LS. The new beta-lactamases. N Engl JMed. 2005;352(4):380-91.

9. Gniadkowski M. Evolution and epidemiology of extended-spec- trum beta-lactamases (ESBLs) and ESBL-producing microorganisms. Clin Microbiol Infect. 2001;7(11):597-608.

10. Perez F, Hujer AM, Hujer KM, Decker BK, Rather PN, Bonomo RA Global challenge of multidrug-resistant Acinetobacter baumannii. Antimicrob Agents Chemother. 2007;51(10):3471-84.

11. Poirel L, Nordmann P. Carbapenem resistance in Acinetobacter baumannii: mechanisms and epidemiology. Clin Microbiol Infect. 2006;12(9):826-36.

12. Lee K, Yong D, Jeong SH, Chong Y. Multidrug-resistant Acinetobacter spp.: increasingly problematic nosocomial pathogens. Yonsei Med J. 2011;52(6):879-91.

13. Shaw KJ, Rather PN, Hare RS, Miller GH. Molecular genetics of aminoglycoside resistance genes and familial relationships of the aminoglycoside-modifying enzymes. Microbiol Rev. 1993;57(1):138-63.

14. Miller GH, Sabatelli FJ, Naples L, Hare RS, Shaw KJ. The most frequently occurring aminoglycoside resistance mechanisms-combined results of surveys in eight regions of the world. The Aminoglycoside Resistance Study Groups. J Chemother. 1995;7 Suppl 2:17-30.

15. Feizabadi MM, Fathollahzadeh B, Taherikalani M, Rasoolinejad M, Sadeghifard N, Aligholi M, et al. Antimicrobial susceptibility patterns and distribution of blaOXA genes among Acinetobacter spp. Isolated from patients at Tehran hospitals. Jpn J Infect Dis. 2008;61(4):274-8.

16. Turton JF, Woodford N, Glover J, Yarde S, Kaufmann ME, Pitt TL Identification of Acinetobacter baumannii by detection of the blaOXA-51-like carbapenemase gene intrinsic to this species. $J$ Clin Microbiol. 2006;44(8):2974-6.

17. Yousefi S, Farajnia S, Nahaei MR, Akhi MT, Ghotaslou R, Soroush $\mathrm{MH}$, et al. Detection of metallo-beta-lactamase-encoding genes among clinical isolates of Pseudomonas aeruginosa in northwest of Iran. Diagn Microbiol Infect Dis. 2010;68(3):322-5.

18. Nemec A, Dolzani L, Brisse S, van den Broek P, Dijkshoorn L. Diversity of aminoglycoside-resistance genes and their association with class 1 integrons among strains of pan-European Acinetobacter baumannii clones. J Med Microbiol. 2004;53(Pt 12):1233-40.

19. Farajnia S, Azhari F, Alikhani MY, Hosseini MK, Peymani A, Sohrabi N. Prevalence of PER and VEB Type Extended Spectrum Betalactamases among Multidrug Resistant Acinetobacter baumannii Isolates in North-West of Iran.Iran JBasic Med Sci. 2013;16(6):751-5.

20. Moniri R, Farahani RK, Shajari G, Shirazi MN, Ghasemi A. Molecular epidemiology of aminoglycosides resistance in acinetobacter spp. With emergence of multidrug-resistant strains. Iran J Public Health. 2010;39(2):63-8.

21. Shahcheraghi F, Abbasalipour M, Feizabadi M, Ebrahimipour G, Akbari N. Isolation and genetic characterization of metallobeta-lactamase and carbapenamase producing strains of Acinetobacter baumannii from patients at Tehran hospitals. Iran J Microbiol. 2011;3(2):68-74.

22. Akers KS, Chaney C, Barsoumian A, Beckius M, Zera W, Yu X, et al. Aminoglycoside resistance and susceptibility testing errors in Acinetobacter baumannii-calcoaceticus complex. J Clin Microbiol. 2010;48(4):1132-8.

23. Nigro SJ, Post V, Hall RM. Aminoglycoside resistance in multiply antibiotic-resistant Acinetobacter baumannii belonging to global clone 2 from Australian hospitals. J Antimicrob Chemother. 2011;66(7):1504-9. 